\title{
Collagenous Fibroma (Desmoplastic Fibroblastoma) of the Neck Presenting with Neurological Symptoms
}

\author{
Farina Fong $\cdot$ Edward Odell $\cdot$ Ricard Simo
}

Received: 24 August 2008/Accepted: 26 November 2008/Published online: 10 December 2008

(C) The Author(s) 2008. This article is published with open access at Springerlink.com

\begin{abstract}
Collagenous fibromas are rare fibrous soft tissue tumours that usually arise in subcutaneous tissue or skeletal muscle at a variety of anatomical sites. These lesions commonly present as painless, slow-growing mobile masses. We describe a unique case of a 41-year-old woman presenting with a posterior neck swelling and longstanding history of severe ongoing pain in the right scapular region, shoulder and neck, weakness of the palmar grip and limited right lateral neck flexion and rotation. A history of trauma to the right neck in adolescence was noted. Histological analysis revealed a paucicellular lesion with spindle and stellate-shaped fibroblasts involving the cervical nerve roots, typical of collagenous fibroma. In a literature search on Medline and Pubmed, we found no reported cases of collagenous fibromas presenting with neurological symptoms. This report highlights the potential of these lesions to present with neurological symptoms due to infiltration of surrounding tissues, and that preceding trauma may contribute to the aetiology.
\end{abstract}

Keywords Desmoplastic fibroblastoma .

Collagenous fibroma - Fibroma - Soft tissue lesion ·

Neck mass $\cdot$ Neurological manifestations

F. Fong $(\bowtie) \cdot$ R. Simo

Department of Otorhinolaryngology, Guy's and St. Thomas'

NHS Foundation Trust, London, UK

e-mail: farina@doctors.org.uk

E. Odell

Department of Oral Pathology, Guy's and St. Thomas' NHS

Foundation Trust, London, UK

\section{Introduction}

The term "desmoplastic fibroblastoma" was first coined by Evans in a report of seven cases published in 1995 to classify a distinctive benign fibrous soft tissue tumour [1]. The tumours are characteristically grossly well circumscribed, with spindle to stellate fibroblasts dispersed in a fibromyxoid or densely fibrous matrix with low mitotic activity. Renamed "collagenous fibroma" a year later by Nielsen et al. [2], collagenous fibromas are rare benign fibroblastic tumours that classically present as non-tender, firm, slowgrowing masses. The largest cohort to date is a case series of 63 patients published by Miettinen and Fetsch [3], elucidating the clinicopathological features of the lesion. Collagenous fibromas occur predominantly in males, with a median age of presentation of 50 years. They most commonly arise in the arm or shoulder [3, 4], but have also been described in the neck [5], back [6], hip [7], extremities [8], parotid [9] and palate [10, 11]. Lesions can typically measure $4 \mathrm{~cm}$ in maximum diameter, although tumours up to $20 \mathrm{~cm}$ in size have been reported [1-3]. The recommended treatment is complete surgical excision [1-13].

\section{Case Report}

A 41-year-old woman presented with a non-tender swelling in the right posterior neck, increasing in size over a period of 2 months. A history of previous significant trauma incurred during adolescence was noted, in which the patient had fallen from a horse and had been kicked in the right side of the neck. Further questioning revealed a 10-year history of severe ongoing pain over the right scapular region, shoulder and neck with no other sensory deficit. Neurological examination demonstrated weakness 


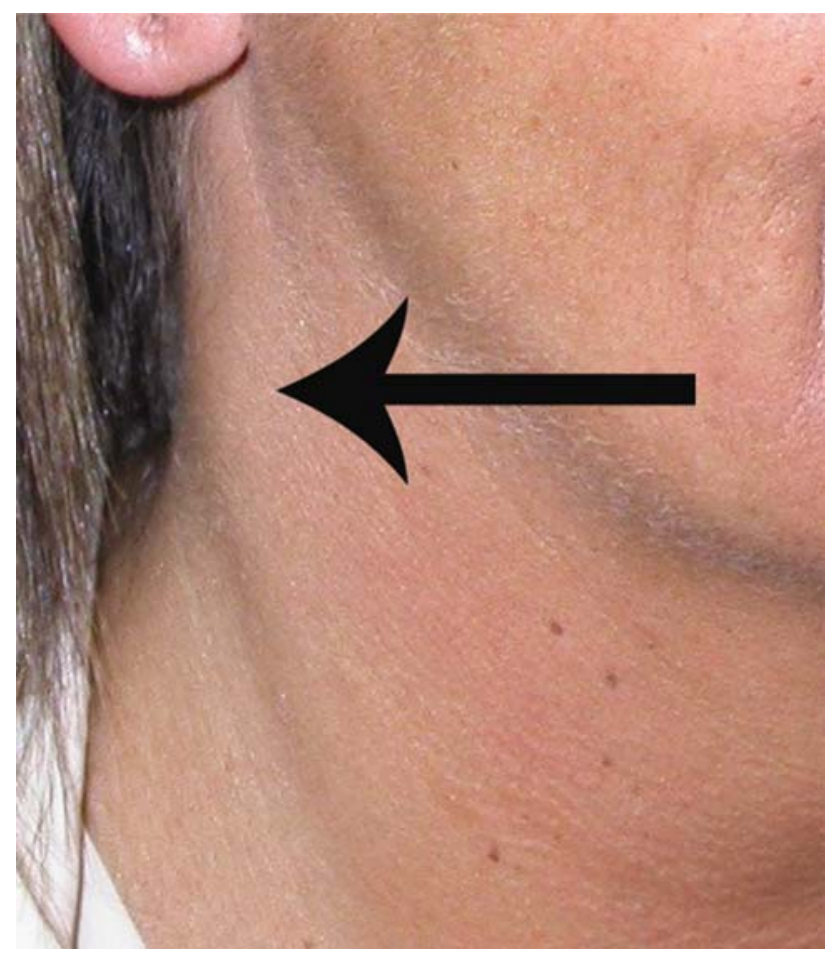

Fig. 1 Clinical appearance of tumour

of palmar grip of the right hand, and limitation of right lateral rotation and flexion of the neck. She had previously been clinically diagnosed with unilateral cervical spondylosis but no radiological evidence was sought at the time. Examination of the neck revealed the presence of a $3 \times 2 \mathrm{~cm}^{2}$ firm non-mobile mass located deep to the posterior border of the right sternocleidomastoid muscle at the level of $\mathrm{C} 3 / \mathrm{C} 4$ (Fig. 1).

Ultrasound scanning showed a hyperechoic mass within the pre-vertebral muscles of the posterior triangle of the neck and fine needle aspiration failed to yield sufficient aspirate for cytopathological analysis. A computerised tomography scan revealed a soft tissue mass arising laterally to the origin of the deltoid muscle, suggestive of a soft tissue sarcoma. Magnetic resonance imaging (MRI) of the neck showed a $3 \times 2 \times 1 \mathrm{~cm}^{3}$ lesion of intermediate T1 and low T2 signal intensity within the right posterior cervical space, that could not be separated from the adjacent scalene and levator scapulae muscles. There was no evidence of cervical spondylosis. A peripheral area of enhancement surrounded the lesion, suggesting a neoplastic or inflammatory process (Fig. 2). An incisional biopsy was recommended by the multidisciplinary head and neck oncology team and the histological analysis confirmed the diagnosis of collagenous fibroma.

Following a discussion by the multidisciplinary head and neck oncology team, a surgical excision of the mass was recommended. This was performed through a transverse cervical incision. The tumour was identified

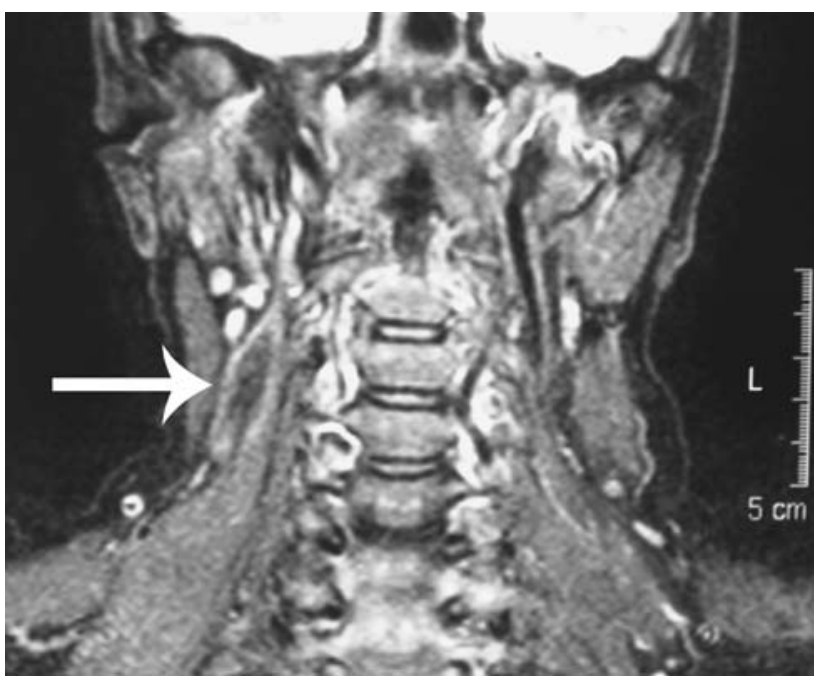

Fig. 2 T2-weighted MRI scan of neck (coronal view). The tumour (arrow) is shown in the right cervical space as an area of low signal intensity with a surrounding region of peripheral enhancement

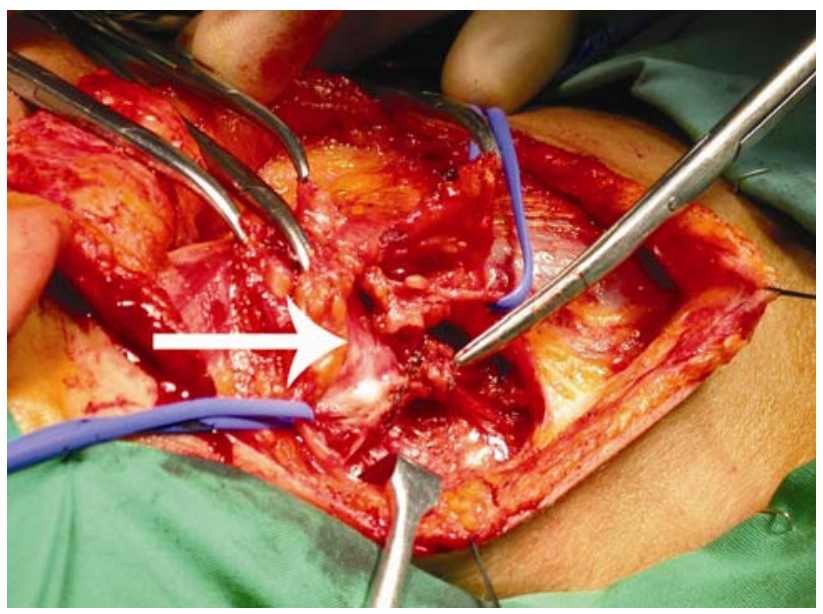

Fig. 3 Intraoperative appearance of tumour. The tumour (arrow) is shown in relation to the right upper cervical plexus (identified by the lower sling) and the right spinal accessory nerve (CN XI) (identified by the upper sling)

intramuscularly, deep to the levator scapulae, scalenus anterior and posterior muscles, involving the upper cervical nerve roots (Figs. 3 and 4). Macroscopically, the tumour was a well-circumscribed firm oval mass, tan-grey in colour with a homogeneous consistency. The resected specimen consisted of mature fat, lymphoid tissue and nerve trunks containing a paucicellular fibrous lesion with spindle to stellate-shaped fibroblasts and a diffuse, permeative architecture mimicking infiltration and extending between and around nerves and muscle bundles. No mitotic figures or nuclear hyperchromasia was seen (Fig. 5). Fibroblasts exhibited a myofibroblastic phenotype on immunocytochemistry, expressing calponin but not smooth muscle actin, S100 protein, CD34 or HMB45. Nerve sheath tumours and 

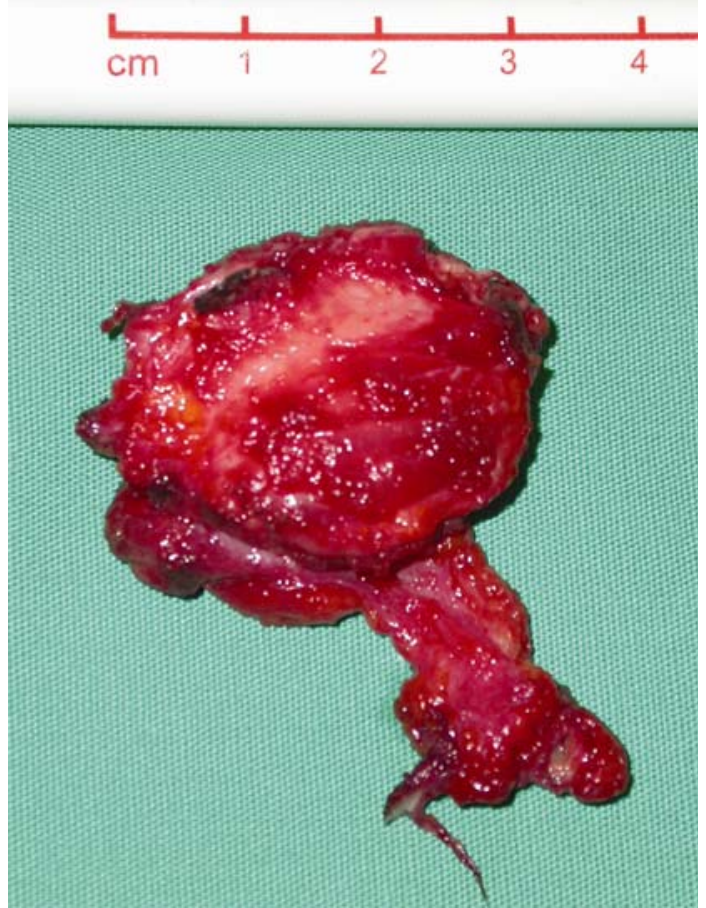

Fig. 4 Resected specimen

solitary fibrous tumour were thus excluded. There were no cytological atypia to suggest a malignant neoplasm. The patient's neurological symptoms improved following surgery and no recurrence was reported at 18-month follow-up.

\section{Discussion}

Since its initial description in 1995, approximately 90 cases of collagenous fibroma have been reported in the literature. As clinicopathological knowledge of these tumours has increased, collagenous fibromas appear a more heterogeneous group of tumours than originally envisaged, as evidenced by their presentation in a variety of anatomical sites. They have even been reported to present as goitre [12] or arise in pre-existing pemphigus vulgaris [13].

Collagenous fibromas characteristically present as slowly enlarging non-tender masses that are noted to increase in size for over a year in approximately one-third of cases [3]. Presentation with ongoing sensorimotor neurological deficit constitutes a rare feature that has not previously been described to our knowledge, despite nerve entrapment being a frequent feature [3]. In this case, the history of trauma to the neck during adolescence following by progressive localised neurological symptoms suggests a substantially longer period of development of this lesion than suggested by the clinically palpable mass. Within the cohort described by Miettinen and Fetsch, there were two cases in which preceding trauma was a feature. However, in both these cases, there was a much shorter period (less than 2 years) between the initial trauma and the development of a palpable tumour [3]. The neurological symptoms in our case are unlikely to be caused by direct nerve injury from the antecedent trauma, because the onset of these symptoms occurred approximately 15 years after the trauma episode and improved after removal of the lesion.

MRI remains the preferred imaging modality at present to delineate collagenous fibromas, which have low signal intensity on $\mathrm{T} 2$-weighted scans ascribed to the low cellularity of these lesions. However, a finding of low T2 signal intensity of a soft tissue tumour on MRI is not unique to collagenous fibromas, and important differential diagnoses cannot be excluded on the basis of this finding. These include neurofibroma, cicatricial fibroma, malignant fibrous histiocytoma, aggressive fibromatosis, calcified masses and soft tissue malignant sarcomas [4].
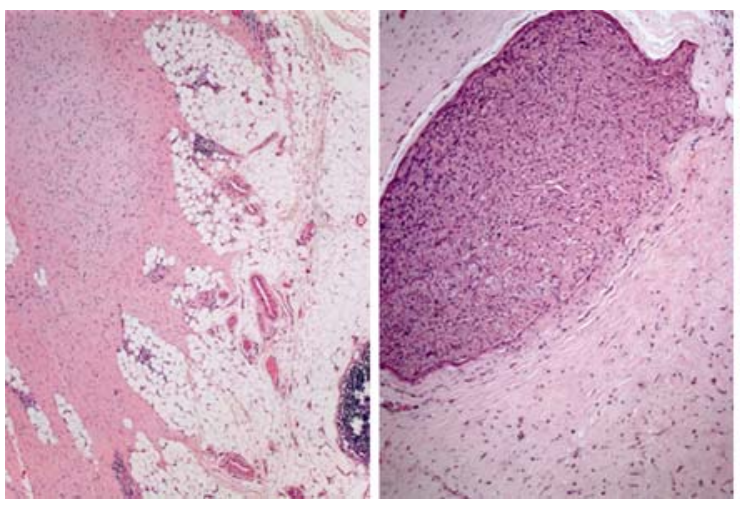

Fig. 5 (left to right): Histological appearance of the tumour (haematoxylin and eosin staining). Panel $1(2 \times)$ showing the margin of the lesion with characteristic sharply defined margin that incorporates fat, muscle and nerve. Panel $2(4 \times)$ showing diffuse cellular fibrous tissue extending around and merging with a large nerve. Panel

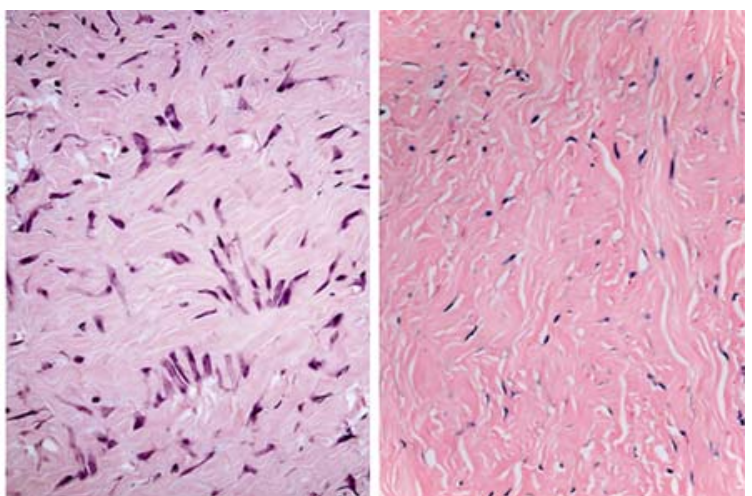

$3(20 \times)$ high power showing a very cellular focus with the typical plump, polygonal, stellate and broad spindled fibroblasts. Panel 4 $(10 \times)$ showing desmoplastic acellular fibrous tissue with dense collagen bundles, forming the bulk of the lesion 
Collagenous fibromas typically infiltrate fat and skeletal muscle, and this has been observed in up to $51 \%$ of cases [3]. Miettinen and Fetsch have described nerve entrapment on a histological level in up to a one-third of cases but have not noted the presence of any neurological symptoms in these patients [3].

Collagenous fibromas may be misdiagnosed as other neoplastic or reactive processes. Principal differential diagnoses include neurofibroma, fibroma of tendon sheath, malignant fibrous histiocytoma, fibromatosis and calcifying fibrous pseudotumour [3, 4, 14]. It is important to differentiate these tumours from other soft tissue lesions, particularly malignant soft tissue tumours due to implications regarding appropriate treatment. This case was fully discussed by the multidisciplinary head and neck oncology team and an incisional biopsy was recommended to obtain tissue for histological diagnosis. The incision was made following surgical oncology principles so that these could easily be extended and excised at the time of the definitive surgery. The recommended treatment of collagenous fibromas is local surgical excision to minimise potential morbidity. There are no reports of local recurrence or metastases to date [1-13, 15], the longest follow-up period being 12 years [15].

Evans postulated that collagenous fibromas might be either neoplasms or reactive conditions [1]. Cytogenetic analysis has revealed the lesion to be clonal and harbour a $\mathrm{t}(2 ; 11)(\mathrm{q} 31 ; \mathrm{q} 12)$ translocation that has also been found in fibromas of the tendon sheath, supporting a neoplastic process[16, 17]. A history of antecedent trauma has been noted in only a few reported cases of collagenous fibroma [3] but it is not clear whether a history of trauma has been actively excluded in all reported cases. Our report reinforces the possibility that fibrous proliferation subsequent to trauma may contribute to the development of these tumours, and that care should be taken to elicit any history of previous trauma that may support diagnosis of this rare tumour.

In summary, we report a presentation of a collagenous fibroma of the neck that presented with a longstanding history of neurological symptoms and previous trauma to the neck. Whilst these tumours classically present with a painless enlarging mass, this report highlights potential presentation with symptoms due to local infiltration of surrounding tissues and suggests that previous trauma may be an underlying aetiological factor.

Open Access This article is distributed under the terms of the Creative Commons Attribution Noncommercial License which permits any noncommercial use, distribution, and reproduction in any medium, provided the original author(s) and source are credited.

\section{References}

1. Evans HL. Desmoplastic fibroblastoma a report of seven cases. Am J Surg Pathol. 1995;19(9):1077-81. doi:10.1097/00000478199509000-00012.

2. Nielsen GP, O'Connell JX, Dickersin GR, Rosenberg AE. Collagenous fibroma (desmoplastic fibroblastoma): a report of seven cases. Mod Pathol. 1996;9:781-5.

3. Miettinen M, Fetsch JF. Collagenous fibroma (desmoplastic fibroblastoma): a clinicopathologic analysis of 63 cases of a distinctive soft-tissue lesion with stellate-shaped fibroblasts. Hum Pathol. 1998;29:676-82. doi:10.1016/S0046-8177(98)90275-1.

4. Walker KR, Bui-Mansfield LT, Gering SA, Ranlett RD. Collagenous fibroma (desmoplastic fibroblastoma) of the shoulder. AJR. 2004; 183:1766.

5. Dagli M, Eryilmaz A, Acar A, Kulacoglu S, Akmansu H. Collagneous fibroma (desmoplastic fibroblastoma). Yonsei Med J. 2004;45:941-3.

6. Jang JG, Jung HH, Suh KS, Kim ST. Desmoplastic fibroblastoma (collagenous fibroma). Am J Dermatopathol. 1999;21:256-8. doi: 10.1097/00000372-199906000-00009.

7. Beggs I, Salter DS, Dorfman HD. Synovial desmoplastic fibroblastoma of hip joint with bone erosion. Skeletal Radiol. 1999;28:402-6. doi:10.1007/s002560050537.

8. Nishio J, Iwasaki H, Nishijima T, Kikuchi M. Collagenous fibroma (desmoplastic fibroblastoma) of the finger in a child. Pathol Int. 2002;52:322-5. doi:10.1046/j.1440-1827.2002.01351.x.

9. Ide F, Shimoyama T, Horie N, Tanaka H. Collagenous fibroma (desmoplastic fibroblastoma) presenting as a parotid mass. J Oral Pathol Med. 1999;28:465-8.

10. Mesquita RA, Okda E, Jorge WA, Cavalcanti de Araujo V. Collagenous fibroma (desmoplastic fibroblastoma) of the palate. Oral Surg Oral Med Oral Pathol Oral Radiol Endod. 2001;91: 80-4. doi:10.1067/moe.2001.109638.

11. Shimoyama T, Horie H, Ide F. Collagenous fibroma (desmoplastic fibroblastoma): a new case originating in the palate. Dentomaxillofac Radiol. 2005;34:117-9. doi:10.1259/dmfr/2242 8083.

12. Summerall WC, Lubin J, Mesko TW. Collagenous fibroma (desmoplastic fibroblastoma): a unique presentation as a goiter in an 88-year-old man. Ann Diagn Pathol. 2000;4:165-9. doi:10.1016/ S1092-9134(00)90040-6.

13. Huang H, Sung M, Eng H, Huang C, Huang W, Chen W. Superficial collagenous fibroma: immunohistochemical, ultrastructural and flow cytometric study of three cases, including one pemphigus vulgaris patient with a dermal mass. APMIS. 2002;110:283-9. doi:10.1034/j.1600-0463.2002.100402.x.

14. Shuto R, Kiyosue H, Hori Y, Miyake H, Kawano K, Mori H. CT and MR imaging of desmoplastic fibroblastoma. Eur Radiol. 2002;12:2474-6.

15. Magro G, Venti C. Childhood desmoplastic fibroblastoma (collagenous fibroma) with a 12-year follow-up. Pediatr Dev Pathol. 1999;2(1):62-4. doi:10.1007/s100249900091.

16. Sciot R, Samson I, Van den Berghe H, Van Damme B, Dal Cin P. Collagenous fibroma (desmoplastic fibroblastoma): genetic link with fibroma of tendon sheath? Mod Pathol. 1999;12:565-8.

17. Bernal K, Nelson M, Neff JR, Nielsen SM, Bridge JA. Translocation $(2 ; 11)(\mathrm{q} 31 ; \mathrm{q} 12)$ is recurrent in collagenous fibroma (desmoplastic fibroblastoma). Cancer Genet Cytogenet. 2004; 149:161-3. doi:10.1016/S0165-4608(03)00298-X. 